\title{
Atypical Familial Mediterranean Fever Complicated with Gastrointestinal Amyloidosis Diagnosed due to Paroxysmal Arthralgia and Intractable Diarrhea, Successfully Treated with Tocilizumab
}

\author{
Erika Aikawa ${ }^{1}$, Toshimasa Shimizu ${ }^{1,2}$, Tomohiro Koga ${ }^{1}$, Yushiro Endo ${ }^{1}$, Masataka Umeda ${ }^{1}$, \\ Tomoko Hori ${ }^{3}$, Junji Irie ${ }^{4}$, Kishio Kuroda ${ }^{5}$, Mizuna Eguchi ${ }^{1}$, Momoko Okamoto ${ }^{1}$, \\ Sosuke Tsuji ${ }^{1}$, Ayuko Takatani ${ }^{1}$, Takashi Igawa ${ }^{1}$, Remi Sumiyoshi ${ }^{1}$, Shin-ya Kawashiri ${ }^{1}$, \\ Naoki Iwamoto ${ }^{1}$, Kunihiro Ichinose ${ }^{1}$, Mami Tamai ${ }^{1}$, Hideki Nakamura ${ }^{1}$, \\ Tomoki Origuchi ${ }^{1,6}$ and Atsushi Kawakami ${ }^{1}$
}

\begin{abstract}
:
A 53-year-old man with recurrent episodes of large joint pain and a low-grade fever at irregular intervals for 16 years developed right knee and ankle arthralgia, watery diarrhea, and abdominal pain. Following an ileum and colon biopsy, he was diagnosed with gastrointestinal amyloidosis. We suspected familial Mediterranean fever (FMF) based on his history and administered colchicine; his symptoms subsequently improved. Thus, he was diagnosed with atypical FMF. After tocilizumab administration, the amyloid deposits disappeared. This case suggests that physicians should consider FMF even in cases with atypical symptoms in order to prevent the progression of amyloidosis and that amyloid deposits can be eliminated by interleukin (IL)-6 inhibition.
\end{abstract}

Key words: Familial Mediterranean fever, amyloidosis, tocilizumab

(Intern Med 58: 1781-1785, 2019)

(DOI: 10.2169/internalmedicine.2277-18)

\section{Introduction}

Amyloid A (AA) amyloidosis is an intractable condition caused by persistent amyloid deposition in multiple organs $(1,2)$. Familial Mediterranean fever (FMF) is an inflammatory condition that can cause AA amyloidosis. FMF is an autoinflammatory disease primarily characterized by a periodic fever and serositis, and $4-5 \%$ of cases are complicated with AA amyloidosis in Japan $(3,4)$. FMF typically involves febrile attacks occurring at regular intervals; however, there are also atypical cases in which a fever occurs at irregular intervals and serositis symptoms are scant, and such atypical symptoms may delay the diagnosis of FMF.

We herein report a case of gastrointestinal amyloidosis (AA type) owing to refractory diarrhea, wherein the diagnosis of FMF was made based on the presence of paroxysmal arthralgia for over a decade.

\section{Case Report}

A 53-year-old Japanese man presented to his previous physician with right knee and ankle arthralgia in May 2017. He had developed watery diarrhea and abdominal pain at the same time, which persisted for several weeks. Lower gastrointestinal endoscopy revealed mucosal edema and ulcers in

${ }^{1}$ Department of Immunology and Rheumatology, Nagasaki University Graduate School of Biomedical Sciences, Japan, ${ }^{2}$ Clinical Research Center, Nagasaki University Hospital, Japan, ${ }^{3}$ Department of Gastroenterology, Nagasaki Harbor Medical Center, Japan, ${ }^{4}$ Department of Pathology, Nagasaki Harbor Medical Center, Japan, ${ }^{5}$ Department of Pathology, Nagasaki University Graduate School of Biomedical Sciences, Japan and ${ }^{6}$ Department of Rehabilitation Sciences, Nagasaki University Graduate School of Biomedical Sciences, Japan Received: October 18, 2018; Accepted: November 28, 2018; Advance Publication by J-STAGE: February 1, 2019 Correspondence to Dr. Toshimasa Shimizu, toshimasashimizu2000@yahoo.co.jp 
A

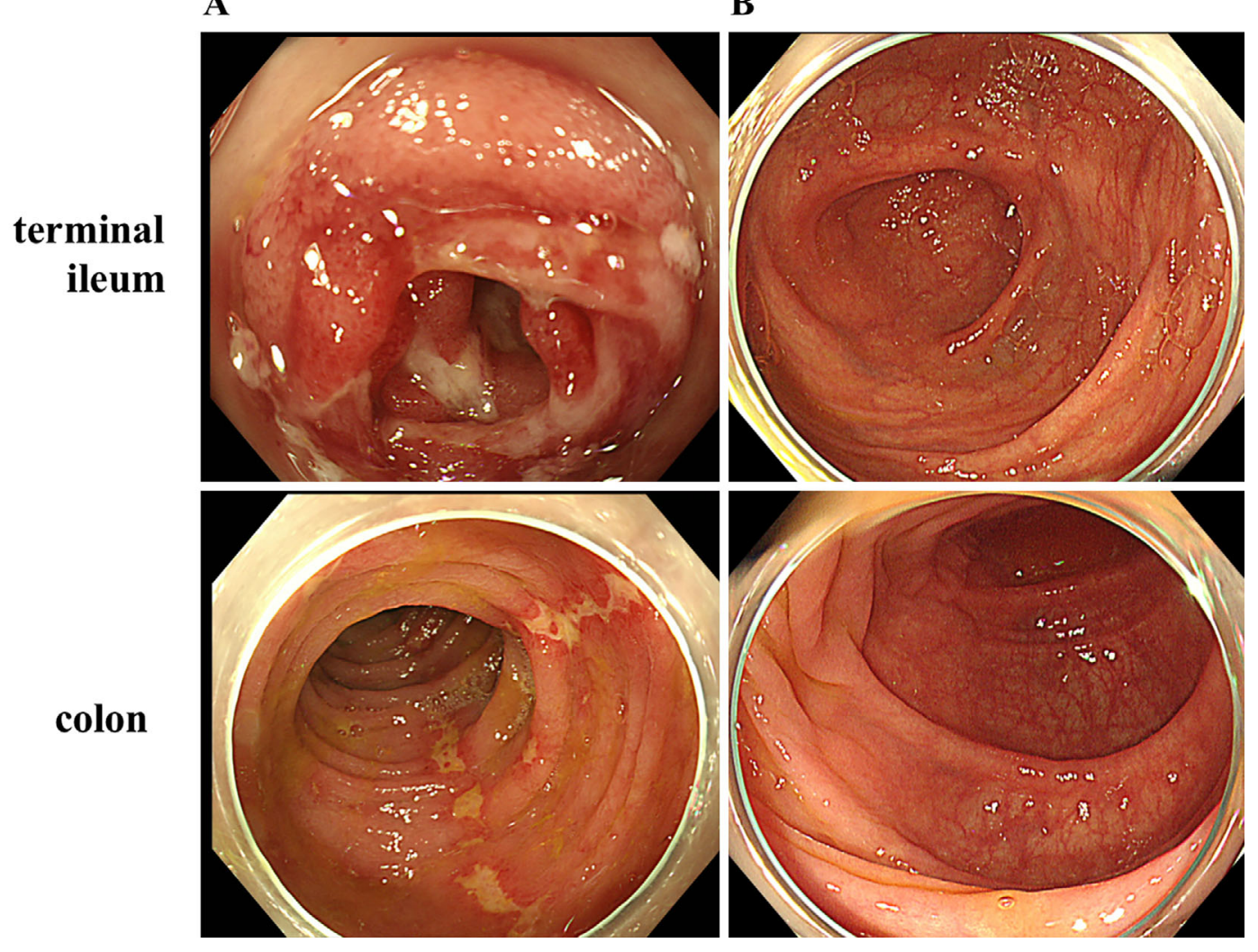

Figure 1. Lower gastrointestinal endoscopy findings. A: Mucosal edema and an ulcer located in the terminal ileum, and irregular longitudinal ulcers from the ascending to descending colon in May 2017. B: Mucosal edema and ulcers improved in November 2017.

the terminal ileum, as well as irregular longitudinal ulcers from the ascending to the descending colon (Fig. 1A). Abdominal computed tomography (CT) did not reveal signs of serositis. Because of the suspicion of inflammatory bowel disease, 10 sites from the ileum to the rectum were biopsied, resulting in findings of AA amyloidosis (Fig. 2A and B). No findings suggestive of inflammatory bowel disease were observed. He was transferred to our department in mid-June 2017 for the further examination and treatment for the AA amyloidosis.

On admission, his diarrhea and abdominal pain persisted, and he continued enteral nutrition as per the recommendations of the previous physician. He had no joint symptoms at that time. His vital signs were as follows: temperature, $36.9^{\circ} \mathrm{C}$; pulse rate, $95 / \mathrm{min}$; blood pressure, $140 / 83 \mathrm{mmHg}$; oxygen saturation, $98 \%$ on room air; and clear consciousness. A physical examination revealed tenderness in the right abdomen and no signs of peritoneal irritation. There was no swelling or tenderness in his joints. No stomatitis or genital ulcers suggestive of Behçet's disease was found. He had no family history of a periodic fever.

Laboratory tests showed the following results: white blood cell (WBC) count 4,700/ $\mathrm{LL}$ (neutrophils 39\%, lymphocytes 48\%), hemoglobin (Hb) $11.5 \mathrm{~g} / \mathrm{dL}$, platelet (PLT) $29.1 \times 10^{4} / \mu \mathrm{L}$, C-reactive protein (CRP) level $0.12 \mathrm{mg} / \mathrm{dL}$, blood urea nitrogen (BUN) $23 \mathrm{mg} / \mathrm{dL}$, creatinine $(\mathrm{Cr}) 1.05$ $\mathrm{mg} / \mathrm{dL}$, uric acid (UA) $8.2 \mathrm{mg} / \mathrm{dL}$, albumin (Alb) $3.2 \mathrm{~g} / \mathrm{dL}$, and an erythrocyte sedimentation rate (ESR) $28 \mathrm{~mm} / \mathrm{h}$; no abnormalities were noted on a urinalysis. The levels of rheumatoid factor (RF) were elevated at $78.4 \mathrm{IU} / \mathrm{mL}$; however, anti-cyclic citrullinated peptide (CCP) antibody were not elevated. Anti-nuclear antibody, myeloperoxidase antineutrophil cytoplastic antibody (MPO-ANCA), and proteinase-3 (PR3)-ANCA were not detected. Two sets of blood cultures and viral markers for hepatitis $\mathrm{B}$ and $\mathrm{C}$ were all negative. Matrix metalloproteinase-3 (MMP-3) was within the standard range at $68.4 \mathrm{ng} / \mathrm{dL}$, whereas serum amyloid A (SAA) was mildly elevated at $11.7 \mu \mathrm{g} / \mathrm{mL}$. No human leukocyte antigen (HLA)-B51 or A26 was detected. Joint radiographs of bilateral hands, fingers, knees, ankles, and toes showed no abnormalities, including no erosion or joint space narrowing suggestive of rheumatoid arthritis (RA), and musculoskeletal ultrasound did not reveal any synovitis in the peripheral joints. Echocardiography demonstrated no abnormalities, and there were no findings suggestive of amyloidosis in the other organs.

When we reconfirmed his medical history, he had experienced recurrent episodes of paroxysmal swelling and tenderness of the elbow, ankle, and knee joints complicated with a low-grade fever at $37^{\circ} \mathrm{C}$ starting around 16 years earlier, with each incident lasting for a few weeks to a month. At that time, he had no abdominal symptoms or skin lesions.

On hospital day 15, he developed swelling and tenderness of the left elbow and a low-grade fever $\left(37^{\circ} \mathrm{C}\right)$, with ele- 

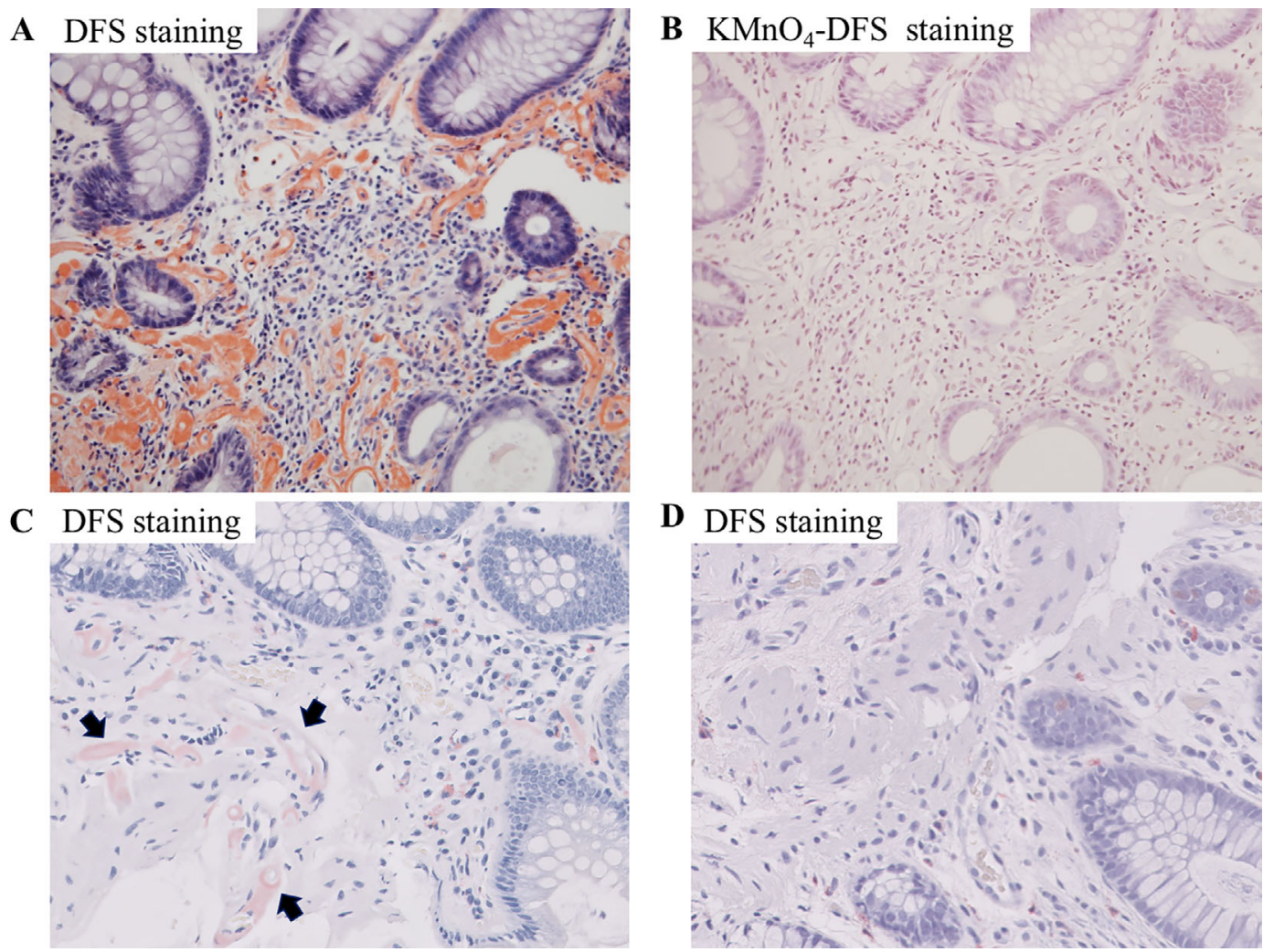

Figure 2. Colon biopsy specimen. A: In the lamina propria, an eosinophilic amorphous material was deposited in the connective tissue and vascular walls, and this material was stained orange-red in May 2017. Direct fast scarlet (DFS) staining, $\times 200$. B: The stain disappeared after $\mathrm{KMnO}_{4}$ oxidation indicated AA amyloidosis findings in May 2017. KMnO4-DFS staining $\times 200$. C: Amyloid depositions persisted in November 2017 (arrows). DFS staining $\times 200$. D: Amyloid depositions completely disappeared on a repeat biopsy in August 2018. DFS staining $\times 200$.

vated inflammatory markers of CRP $(3.73 \mathrm{mg} / \mathrm{dL})$ and SAA $(392.7 \mu \mathrm{g} / \mathrm{mL})$. Since the history of paroxysmal arthralgia and fever suggested FMF, we introduced colchicine at a dose of $1 \mathrm{mg} /$ day as diagnostic treatment. The arthralgia and fever resolved rapidly, and SAA levels dropped to $6.7 \mu \mathrm{g}$ / $\mathrm{mL}$ five days after the initial colchicine administration. Given these symptoms and his response to colchicine, he was diagnosed with FMF based on the Tel Hashomer criteria for incomplete-type FMF (atypical FMF) (5). In addition, an $M E F V$ genetic analysis (exons 1, 2, 3, and 10) revealed multiple heterozygous mutations at E84K in exon 1 and at E148Q in exon 2 (Fig. 3).

His abdominal symptoms, including diarrhea and abdominal pain, also improved and did not worsen again after resuming food intake. Because gastrointestinal amyloidosis was already observed, we began to administer $162 \mathrm{mg}$ subcutaneous tocilizumab every 2 weeks since it was expected to be an effective treatment for AA amyloidosis on hospital day 30 after obtaining the patient's informed consent. Subsequently, a fever and arthralgia were no longer observed, and the findings of lower gastrointestinal endoscopy also improved in November 2017 (Fig. 1B); however, amyloid deposition in the ileum and colon persisted (Fig. 2C). Although colchicine administration was stopped because of alopecia in January 2018, remission has been maintained by tocilizumab therapy. In addition, the amyloid deposition in the ileum and colon disappeared in August 2018 (Fig. 2D)

\section{Discussion}

AA amyloidosis is a type of organopathy that occurs when an inflammatory disorder causes amyloid production and deposition in organs. Amyloid deposits are observed in the gastrointestinal tract, heart, and kidneys and can cause organopathy with a poor prognosis (2). RA is the most frequent causative inflammatory disorder of AA amyloidosis, while FMF was found to account for $5 \%$ of cases in a cohort in the UK (1).

FMF is a type of autoinflammatory disease characterized by a periodic fever and serositis. Typical cases of FMF present with a paroxysmal fever of at least $38^{\circ} \mathrm{C}$ and serositis lasting from 12 to 72 hours. However, there are also atypical cases that differ in the duration and temperature of the fever and have incomplete attacks of serositis (5). Indeed, it $43 \%$ of FMF cases in Japan are reportedly atypical FMF (6). Compared with typical FMF, atypical FMF has been characterized by a higher age at onset, longer fever duration, less abdominal and thoracic pain caused by peritonitis and pleuritis, higher frequency of arthritis and myalgia, and lower frequency of a family history of FMF (6). 


\section{Exon 1}

$\mathrm{E} 84 \mathrm{~K}$

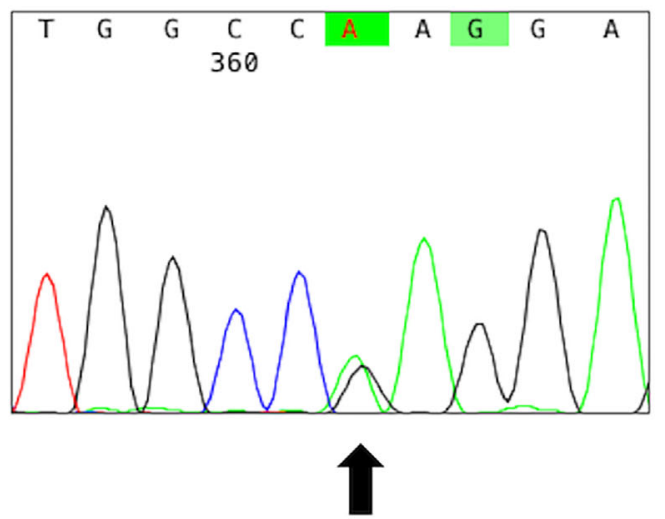

c. $250 \mathrm{G}>\mathrm{A}$

\section{Exon 2}

E148Q

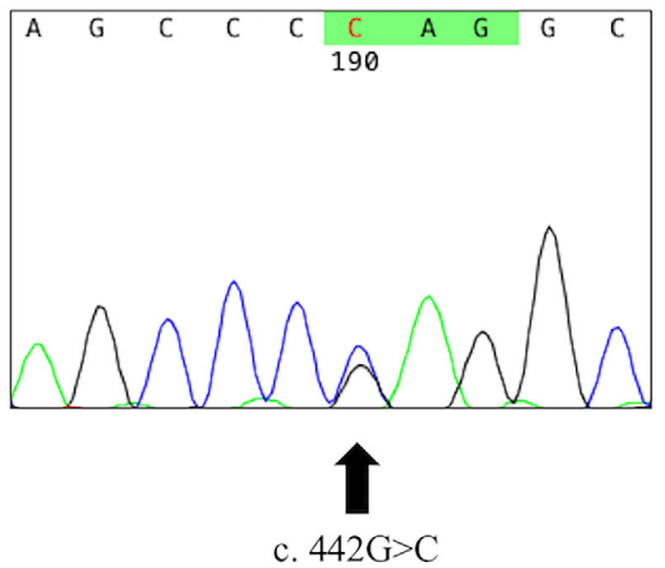

Figure 3. A sequencing analysis of the $M E F V$ gene. DNA sequencing shows a compound heterozygous mutation at E84K in exon 1 and at E148Q in exon 2 of the MEFV gene.

In Japan, AA amyloidosis complicates approximately 4$5 \%$ of FMF cases, which is a lower rate than in the Mediterranean region (13\% of cases in Turkey and $27 \%$ of cases in Israel are complicated by AA amyloidosis) $(3,4)$. Whether typical or atypical, FMF is considered to have no effect on the complications of AA amyloidosis. The presence of an M694V mutation in exon 10 of the $M E F V$ gene and Armenian, Turkish, or Arab race are factors that increase the risk of complications in AA amyloidosis (7). Our patient did not have these risk factors; however, the risk factors for AA amyloidosis complication also include the disease duration (7). FMF patients complicated with AA amyloidosis reportedly show a delayed diagnosis compared to those without such a complication (8). The typical time to the diagnosis from the onset in Japanese FMF patients is approximately nine years (3), and this increases to $20.1 \pm 4.5$ years among cases complicated with AA amyloidosis (9). Our patient was diagnosed with atypical FMF 16 years from the initial onset, suggesting that a delay in the diagnosis was one of the causes of the complication with AA amyloidosis. Given that the average duration of underlying disease until complication with AA amyloidosis has been reported to be 17 years, the early diagnosis of FMF is important (1).

Colchicine is the drug of choice for the treatment of FMF, being shown to be effective in approximately 92\% of cases $(6,10)$. Colchicine suppresses FMF attacks and is thus effective in preventing amyloidosis deposition (11). However, the therapeutic effects on AA amyloidosis are still unclear. Tocilizumab, which is an interleukin (IL)-6 receptor monoclonal antibody, was recently shown to be effective on RA complicated with AA amyloidosis, resulting in the disappearance of amyloid deposition from a histological perspective $(12,13)$. In addition, several reports have indicated that tocilizumab can improve AA amyloidosis complicating FMF (14-17). Indeed, colchicine and tocilizumab treatment not only prevented attacks but also resolved amyloid deposition in our patient. However, the lower gastrointestinal endoscopy findings were atypical for gastrointestinal amyloidosis. Previous reports have noted that the abdominal lesions of FMF can sometimes resemble inflammatory bowel disease, and FMF abdominal lesions were also observed in the mucus membrane lesions in our patient (18).

In conclusion, we encountered a patient with FMF complicated with gastrointestinal amyloidosis that was diagnosed as a result of paroxysmal arthralgia, a low-grade fever, and intractable diarrhea. Our patient had atypical symptoms of FMF, and the diagnosis and treatment were delayed, resulting in the development of AA amyloidosis. Atypical symptoms can be the reason for a delayed diagnosis of FMF; thus, physicians should consider a diagnosis of FMF even in cases with atypical symptoms, such as irregular paroxysmal arthralgia and a low-grade fever. In addition, IL-6 inhibitor treatment for FMF can not only prevent attacks but also resolve AA amyloidosis.

The authors state that they have no Conflict of Interest (COI).

Erika Aikawa and Toshimasa Shimizu contributed equally to this work.

\section{References}

1. Lachmann HJ, Goodman HJ, Gilbertson JA, et al. Natural history and outcome in systemic AA amyloidosis. N Engl J Med 356: 2361-2371, 2007.

2. Merlini G, Bellotti V. Molecular mechanisms of amyloidosis. N Engl J Med 349: 583-596, 2003.

3. Migita K, Uehara R, Nakamura $Y$, et al. Familial Mediterranean fever in Japan. Medicine (Baltimore) 91: 337-343, 2012.

4. Migita K, Izumi Y, Jiuchi Y, et al. Familial Mediterranean fever is no longer a rare disease in Japan. Arthritis Res Ther 18: 175, 
2016.

5. Livneh A, Langevitz P, Zemer D, et al. Criteria for the diagnosis of familial Mediterranean fever. Arthritis Rheum 40: 1879-1885, 1997.

6. Migita K, Agematsu K, Yazaki M, et al. Familial Mediterranean Fever: genotype-phenotype correlations in Japanese patients. Medicine 93: 158-164, 2014.

7. Touitou I, Sarkisian T, Medlej-Hashim M, et al. Country as the primary risk factor for renal amyloidosis in familial Mediterranean fever. Arthritis Rheum 56: 1706-1712, 2007.

8. Tunca M, Akar S, Onen F, et al. Familial Mediterranean fever (FMF) in Turkey: results of a nationwide multicenter study. Medicine (Baltimore) 84: 1-11, 2005.

9. Migita K, Agematsu K. Clinical aspects of Familial Mediterranean fever. Nihon Rinsho Men'eki Gakkai kaishi (Jpn J Clin Immunol) 34: 355-360, 2011 (in Japanese, Abstract in English).

10. Ozen S, Demirkaya E, Erer B, et al. EULAR recommendations for the management of familial Mediterranean fever. Ann Rheum Dis 75: 644-651, 2016.

11. Livneh A, Zemer D, Langevitz P, Laor A, Sohar E, Pras M. Colchicine treatment of AA amyloidosis of familial Mediterranean fever. Arthritis Rheum 37: 1804-1811, 1994.

12. Albrecht K, Albert C, Lange U, Muller-Ladner U, Strunk J. Different effects of local cryogel and cold air physical therapy in wrist rheumatoid arthritis visualised by power Doppler ultrasound. Ann Rheum Dis 68: 1234-1235, 2009.

13. Inoue $\mathrm{D}$, Arima $\mathrm{H}$, Kawanami $\mathrm{C}$, et al. Excellent therapeutic effect of tocilizumab on intestinal amyloid a deposition secondary to active rheumatoid arthritis. Clin Rheumatol 29: 1195-1197, 2010.

14. Yilmaz S, Cinar M, Simsek I, Erdem H, Pay S. Tocilizumab in the treatment of patients with AA amyloidosis secondary to familial Mediterranean fever. Rheumatology (Oxford) 54: 564-565, 2014.

15. Hamanoue S, Suwabe T, Hoshino J, et al. Successful treatment with humanized anti-interleukin-6 receptor antibody (tocilizumab) in a case of AA amyloidosis complicated by familial Mediterranean fever. Mod Rheumatol 26: 610-613, 2016.

16. Serelis J, Christaki S, Skopouli FN. Remission of nephrotic syndrome due to AA-amyloidosis, complicating familiar Mediterranean fever, with tocilizumab. Clin Exp Rheumatol 33: S169, 2015.

17. Koga T, Migita K, Kawakami A. Biologic therapy in familial Mediterranean fever. Mod Rheumatol 26: 637-641, 2016.

18. Arasawa S, Nakase H, Ozaki Y, Uza N, Matsuura M, Chiba T. Mediterranean mimicker. The Lancet 380: 2052, 2012.

The Internal Medicine is an Open Access journal distributed under the Creative Commons Attribution-NonCommercial-NoDerivatives 4.0 International License. To view the details of this license, please visit (https://creativecommons.org/licenses/ by-nc-nd/4.0/).

(C) 2019 The Japanese Society of Internal Medicine Intern Med 58: 1781-1785, 2019 